Revue d'histoire de l'Amérique française

DAS REVUE D.HISTOIRE DE L'AMÉRIQUE FRANÇAISE

\title{
Inventaire des biens de René-Louis Chartier, sieur de Lotbinière (Québec, 13-17 juin 1701)
}

\section{André Vachon}

Volume 21, numéro 4, mars 1968

URI : https://id.erudit.org/iderudit/302726ar

DOI : https://doi.org/10.7202/302726ar

Aller au sommaire du numéro

Éditeur(s)

Institut d'histoire de l'Amérique française

ISSN

0035-2357 (imprimé)

1492-1383 (numérique)

Découvrir la revue

Citer ce document

Vachon, A. (1968). Inventaire des biens de René-Louis Chartier, sieur de Lotbinière (Québec, 13-17 juin 1701). Revue d'histoire de l'Amérique française, 21(4), 803-824. https://doi.org/10.7202/302726ar d'utilisation que vous pouvez consulter en ligne. 


\section{DOCUMENTS INÉDITS}

\section{INVENTAIRE DES BIENS DE RENE-LOUIS CHARTIER, SIEUR DE LOTBINIERE (Québec, 13.17 juin 1701)*}

Issu d'une lignée dont la noblesse remontait au début du $\mathrm{XV}^{\mathrm{e}}$ siècle, fils de Louis-Théandre Chartier de Lotbinière et d'Elizabeth Damours, René-Louis Chartier fut baptisé à SaintNicolas-des-Champs de Paris le 14 novembre 1641. René-Louis et sa famille débarquèrent à Québec le 13 octobre 1651, en compagnie de Jean de Lauson, leur parent, qui venait remplir les fonctions de gouverneur général.

Louis-Théandre Chartier ${ }^{1}$, protégé par Lauson, ne tarda pas à jouer dans la colonie un rôle judiciaire: procureur fiscal (1651-1656) et lieutenant général civil et criminel (1656-1663) de la Sénéchaussée de Québec, procureur général intérimaire du Conseil souverain (1664-1666), Chartier devint, en 1666, le premier lieutenant général de la Prévôté de Québec récemment établie. Il démissionna en 1677 pour rentrer en France, laissant au pays son fils René-Louis et sa fille Marie-Françoise, épouse de Pierre de Joybert de Soulanges et de Marson, commandant en Acadie. Il y mourut peu avant 1690.

René-Louis avait étudié au Collège des Jésuites de Québec. En 1670, âgé de moins de trente ans, il était entré à son tour dans les emplois: substitut du procureur général du Conseil souverain de 1670 à 1674, conseiller de 1674 à 1677, il succéda à son père comme juge de la Prévôté à l'automne de cette dernière année. Estimé pour sa compétence, son zèle et son intégrité, il tint cette charge jusqu'en 1703, alors qu'il fut nommé premier conseiller du Conseil supérieur. Il mourut en fonction le 3 juin juin 1701.

*Archives judiciaires de Québec, Minutier de Louis Chambalon, 13

1 Pour une biographie complète de ce personnage, voir André Vachon, "Chartier de Lotbinière, Louis-Théandre", dans le Dictionnaire biographique du Canada, I: 207s. 
1709. Carrière impressionnante que la sienne: un des principaux officiers de justice de la colonie, bientôt quatrième personnage de la hiérarchie canadienne, Chartier fut de surcroît subdélégué de l'intendant à plusieurs reprises et agent général de la colonie, tout en agissant comme officier de milice de 1666 à 1690 au moins.

Le 24 janvier 1678, René-Louis Chartier avait épousé MarieMadeleine Lambert, fille de feu Eustache Lambert, qui avait été un commerçant en vue de Québec. De cette union naquirent dix enfants, dont six survécurent à leur mère, décédée en novembre 1695. En secondes noces, Chartier épousa, le 16 mai 1701, Françoise Jachée, veuve d'Antoine Gourdeau, un officier de la ferme du roi. C'est peu après ce mariage, en juin 1701, que les notaires Louis Chambalon et Charles Rageot procédèrent à l'inventaire des biens de la communauté qui avait existé entre Chartier et Marie-Madeleine Lambert.

Cet inventaire, que nous présentons ici, offre un grand intérêt pour l'historien, d'autant que l'on n'a peut-être jamais publié de semblables documents relatifs aux officiers de justice. On s'est en général limité jusqu'ici aux gouverneurs, aux intendants, aux évêques du régime français, et plus récemment à quelques commerçants. Or l'inventaire des biens de Chartier de Lotbinière permet de constater le train de vie de cet officier de justice, de soupconner ses habitudes socio-économiques, de pénétrer dans le cadre intime de sa vie familiale, en plus d'évaluer assez exactement sa fortune. Par ailleurs, la description minutieuse de ses papiers apporte des renseignements tout à fait inédits sur la carrière de ce représentant de la noblesse coloniale ${ }^{2}$.

ANDRÉ VACHON

Université Laval

[p. 1]

13. Juin 1701.

\section{INVENTAIRE DE MONSIEUR DE LOTBINIERE LIEUTENANT GENERAL}

L an mil sept Cents Un Le Treiziesme jour de juin [mil] deux heures de Releves a la requeste de Monsieur M.re René Louis Chartier escuyer Seigneur de lotbinière Conseiller du Roy et Son lieutenant general de la prevosté et admirauté de quebec Tant En son nom a cause de la Communauté de biens qui a esté Entre luy et deffunte dame Marie Magdeleine Lambert

2 Noter que les mots raturés par les notaires sont placés entre crochets; que les renvois sont intégrés au texte, mais en italique; et que nos interventions sont entre parenthèses. 
vivante $\mathrm{Sa}$ première épouze que comme Tuteur Des Enfants mineurs Issus de leur mariage Eslu a lad. charge par acte Expedié par devant monsieur le lieutenant particulier de La prevosté de cette d. ville en datte du neuf de ce mois et an, En presence de pierre becart Escuyer Seigneur de Grandville Lieutenant d'une Compagnie dans les trouppes du detashement de la marine Entretenüe en Ce pays Subrogé tuteur desd. mineurs esleu a lad. Charge par le mesme acte A Esté Pour La Conservation des droits desd. mineurs et de quy Il apartiendra par nous louis Chambalon et Charles Rageot notaires Royaux en la prevosté dud. quebec Soubzignes y Residants proceddé a l'inventaire et description des biens meubles, Ustancilles, argent monnoyée et non monnoyée dettes actives et passives, Titres, papiers et autres Enseignements dependant de la Communauté qui a esté entre mond. Sieur Le lieutenant et lad. deffunte dame lambert sa premiere espouse et quy Sont a Separer Entre luy et Sesd. Enfants mineurs; par luy representés et mis En Evidence en la maison a luy apartenant Size en Cette haute ville de quebec ruë Saint louis, apres declaration qu'il a faite par le serment quil a Justice de les representer toutes sur les peines de l'ordonnance de laquelle Il a une parfaite Connoissance; lesquels meubles et Ustencilles ont esté prisées et Estimes par Joseph prieur huissier audiancier en lad. prevosté faisant La fonction de juré priseur vendeur de biens de Justice en cette ville qui a promis den faire la prisée et estimation a $\mathrm{La}$ Juste valeur y Comprise la Cruë eu egard a la qualité et valeur de Chaque Chose au taux present; a laquelle prisée et estimation Il a procedé ainsy quil ensuit, et ont mesd. Sieurs Le lieutenant general, de grand-ville, huissier priseur et notaire Signé

$\mathrm{R}$ L Chartier

De Granville

de Lotbinière

Prieur

Rageot

Chambalon

[p. 2]

Premierement,

Suit ce qui S'est Trouvé dans la salle En Entrant dans lad. maison servant de Cuisine quand a present; et dans une depance au Costé d'ycelle;

Une Vieille Cramaillere estimée Cinquante sols cy

Une paire de petit ou moyens Chenets estimez sept Livres

Une paire de Tenailles et une pelle a feu estimez ensemble à Six Livres cy

Deux hastiers de fer estimez a dix livres

Une vieille petite bassinoire de Cuivre jaune Apiessée estimée Cinquante sols cy

Une vieille passoire de Cuivre toute uzée estimée trente sols

Une vieille petite tourtiere de cuivre rouge apiessée estimée à trente sols cy

Une Chaudière de Cuivre Rouge d'environ Cinq seaux apiessée estimée dix huit livres

Une poislonne de Cuivre Rouge étamée a deux anses de fer apiessée de trois pieces de Cuivre Jaune estimée cinq Livres

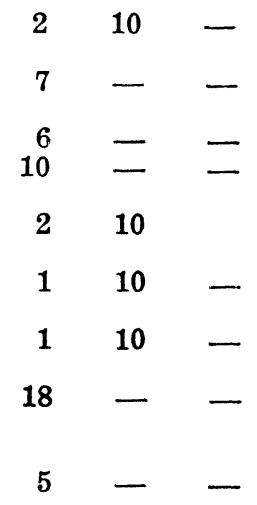


Un poislon de cuivre estimé vingt Sols estant persée Une autre petite poislonne de Cuivre Rouge anses de mesme matiere estimée Cinq Livres cy

Une Chaudiere de Cuivre Rouge denviron deux seaux estimé neuf livres

Deux sceaux ferrez neufs estimez dix livres

Une broche a rôtir cassée en deux morceaux estimée quinze sols

Un autre petite broche a rostir estimée vingt cinq sols Un gril de fer estimé cinquante sols

Un Rechaud de Cuivre jaune pieds de fer estimé cinquante sols

Une vieille lèchefritte de cuivre rouge Toute uzée qui n'est presque que pour mitraille estimée trente sols Une marmitte de fer Sans Couvercle estimée Cinq livres Un pot de fer avec son couvercle de Cuivre rouge estimé quatre livres dix sols cy

Une moyenne marmite qui a un pied Racommodé estimée avec Une autre petite marmite [de fer] aussy de fer d'environ Une pinte estimée Ensemble cinquante sols [p. 3]

Deux couteaux de Cuivre a hacher de la viande estimez ensemb. ${ }^{e}$ Cinq livres

Trois petites fers a flasquer estimez a trente sols piece fait ensemble quatre livres dix sols

Un mortier de fonte avec son pilon Cassé par la moistié pezant Environ Onze livres cy - estimé treize livres cy Un petit flau de fer d'holande avec deux mechants petits plataux de cuivre l'un rouge et lautre jaune estimé Cinq livres

Un chandelier de Cuivre a pied carré avec une paire de mouchette et un porte mouchette estimé ensemble trois livres dix sols

Trois vieux flambeaux destin cassez ressoudez et vieux estimez ensemble a trois livres quinze sols

Trois autres petits chandelliers destin a petite bougie pour servir dans un cabinet estimes ensemble trente sols Une Caffetiere de fer blanc estimée quinze sols

Un petit crochet de fer a pendre de la viande estimé quarante sols

Trois grands plats, quatre Mazarines ou petits plats et dix huit assiettes pezant ensemble Cinquante trois livres a vingt huit sols la livre soit en tout la somme de soixante quatorze livres quatre sols

Un bassin a laver, deux moyens plats, trois ditto petits, trois mazarines, dix huit assiettes et quatre colliers de maure Letout de vieil estin qui n'est propre qu'a Refondre pezant soixante deux livres et demye a dix sols la livre soit la somme de

Une vieille aiguiere sans couvercle et un pot de vieil estin qui ne sont aussy propre qua Refondre estiméez ensemb.e sur le pied de dix sols la livre a la somme de trois livres quinze sols

Un demy art et un petit moutardier destin estimé qua-

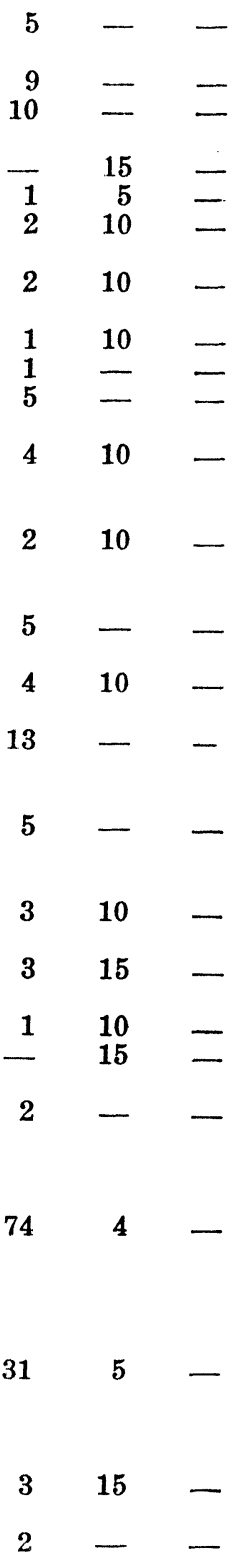
rante sols les deux 
Une Seringue garnye avec son Estuy estimée Sept Livres

Six Plats de fayance, quatorze assiettes, et onze pettites pieces (?) a mettre des Confitures le tout façon de [p. 4]

porcellenne Le tout estimé Ensemble a la Somme de Vingt Livres huit sols

Six assiettes de terre façon de fayance estimées ensemble trente sols

Un petit pot a leau, un huislier, et un petit vinaigrier le tout de fayance estimé ensemble a trente six sols cy Un mortier de pierre du pays avec son pillon de bois estimé Six livres cy

Une mechante poisle a frire [estimée] de nulle valeur estant toute uzée, un mot raturé a cet article ne vault; Une vieille lanterne de fer blanc garnie de corne estimée quarante sols cy

Douze flacons de verre sans bouchons dix de Cinq chopines pieces et deux de trois chopines estimez L'un pour lautre a quinze sols piece cy

Dix sept aunes de moquette neuve a Sept Livres dix sols L'aune fait la somme de cent vingt Sept Livres dix sols Deux milliers de petites Glands (?) dorez a trois Livres dix sols le millier fait la somme de

quatre douzaines de petit Galon faux or a cinquante sols La douzaine fait la somme de dix livres cy

Un bassin destin de Commodité estimé trois livres

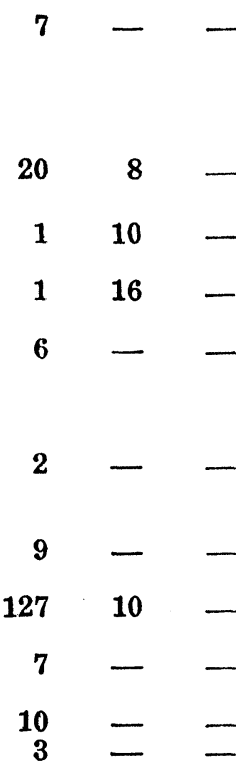

Et attendu quil est pres de Six heures nous avons Remis La Continuation de ced. Inventaire a mercredy prochain Sept heures du matin; et Sont tous les meubles cy dessus Inventoriez demeurez es mains et a la garde de Mond.sieur Le lieutenant general qui S'en est volontairement Chargé du Consentement dud. Sieur de grandville subrogé tuteur quy ont avec Led. prieur huissier present et no. ${ }^{\text {res }}$ Signé;

RL Chartier de Lotbinière

Prieur

Rageot
De Granville

\section{Chambalon}

Et advenant Le quinziesme Dud. mois et an dix heures du matin a La [p. 5] requisition de Mond. sieur Le lieutenant general en presence dud. Sieur De grand-ville subrogé tuteur desd mineurs a esté par lesd. notaires procedé à la Continuation dud. Inventaire ainsy quil Ensuit;

Suitte de ce qui sest trouvé dans Lad. Salle servant de Cuisine et dans Lad. despense quy est au Costé d'ycelle;

Une Grande Tourtiere de Cuivre Rouge étamée estant sans Couvercle vicié et persé estimée dix livres Cinq péroquets de bois de noyer de france garnies de serge verte Lad. garniture entierement uzée [estimée] et un dont le dossié est cassé estimez ensemble a Trente sols piece fait la somme de [neuf livres] sept livres dix sols cy

10 
Le mot Cinq a larticle cy dessus aprouvé pour bon Et les mots neuf livres raturez ne valent;

Un grand Coffre de bois de pin fermant a clef avec une mechante serrure estimé a la somme de six livres cy Une grande Armoire de bois de merisier a deux batants fermant a Clef par une serrure estimée trente livres cy

Dans laquelle S'est trouvé ce qui Suit;

Douze nappes de differentes tailles de chanvre plus de demye uzées d'environ une aune un quart piece à vingt sols piece fait

Le mot douze a larticle cy dessus approuvé pour bon, Dix huit serviettes de toille de chanvre aussy plus de demy Uzée estimees huit sols piece l'une pour lautre qui reviennent a

Vingt quatre autres petites serviettes de toile de Chanvre fort uzées estimées a six sols piece

Un moyen pot de fayance façon de porceleine avec son Couvercle a Confiture estimé vingt sols

deux petits pots a leau et dix autres moyens et petits a confiture de fayance de differentes especes estimées ensemble quatre livres dix sols cy

Une bouteille de Cristal d'environ trois Chopines estimée trois Livres

Un flaçon de vitre de Cinq Chopines avec son bouchon de plomb estimé vingt cinq sols

Six petits flacons ronds de demy art piece a liqueurs a quatre sols piece fait

[p. 6]

Une petite tasse de cristal a boire liqueurs avec son estuy de cuir estimée quinze sols cy

Un vieux coffre bahu garny de Cuir et petites tout rompu fermant a clef estimé dix livres cy

Dans lequel s'est trouvé;

huit linceulx de toille de chanvre d'environ cinq aune et demye piece demy uzés Estimez L'un pour Lautre a cinq Livres dix sols piece ce qui fait ensemble la somme de

Cinq autres linceulx de mesme toille d'environ Cinq aune piece persés, rapiessez et uzes estimez l'un pour lautre a quarente sols piece fait la somme de

Deux autres petits draps ou linceulx de toille de Chanvre presque Uzees et persees estimez quarante sols piece cy

Deux autres linceulx de toille de roüan de Cinq aune et demye piece Estimez a douze livres piece

Un autre petit drap de mesme toille d'environ trois aunes et demye estimé huit livres cy

Onze grandes serviettes de toille de Chanvre fine a barre bleuë estimée avec la nappe de mesme toille de trois aune et demye de toille a la somme de vingt deux livres

Douze serviettes de toille oeuvrée fine avec leur nappe demye Uzees Estimée ensemble a la somme de vingt livres cy

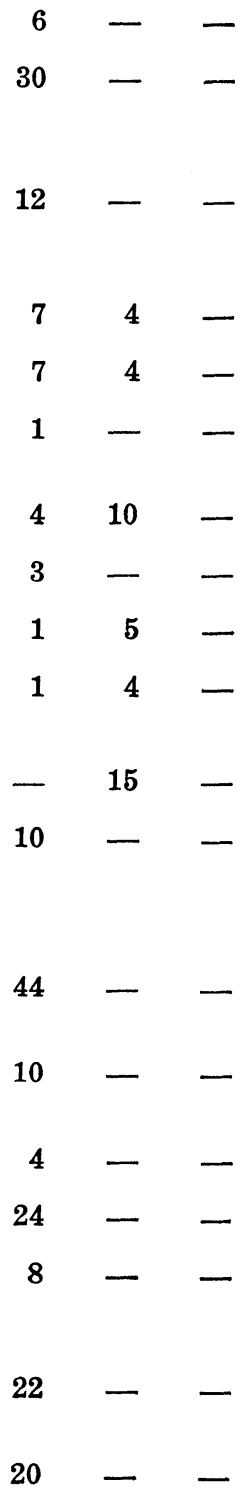


Une autre grande nape de mesme toille fine oeuvrée [est] plus de demye uzée estimée quatre livres dix sols Une autre petite nape et sept serviettes de mesme toille assez fine estimée ensemble treize livres

Deux autres [pet] Nappes de buffet estroittes de moyenne Toille oeuvrée estimée estant plus de demy uzée a trois Livres piece

Une serviette de toille de Chanvre my uzée estimée dix sols

Une mechante petite Table Carrée de bois de pin Avec son tiroüer sans façon avec un vieux tapy vert estimée à quatre livres dix sols cy

Quatre vieux morceaux de tapisserie de bergame presque Uzée et apiessée estimez a la somme de dix livres Une vieille moyenne Table auvalle de bois de pin Cassée avec son pied pliant estimée quarante cinq sols

Un marteau brochoir estimé vingt cinq sols

\section{[p. 7]}

Suit Ce qui C'est trouvé dans La Chambre quy est au Costé de lad. Salle servant de Cuisine, qui a veuë sur le Jardin

Une paire de petits Chenets de fer garnis de pommes et figures de Cuivre estimez quinze livres cy

Un vieux gueridon de merisier estime vingt sols

Une épinette avec son pied de bois de merisier tourné proprem. ${ }^{t}$ Estimée Soixante dix Livres

Douze Chezes de bois de merisier et un fauteuil de mesme bois Le tout trouvé garny de Sauge Estimé Ensemblé a la somme de Cent vingt livres cy

[Ne sera fait au]

Une vieille Cheze caquetoire garnye de broquatelle ou moquette rompuë estimée Cinquante sols

Un buffet carré servant de table a deux pannaux fermant a clef de bois de merisier estimé quinze livres

Un Grand miroüer de vingt poulces de Glace de hauteur sur seize de large cadre de bois plaque de cuivre Estimé quarante cinq livres cy

Un autre petit miroüer de toillette tout de glace de dix à douze poulces de Glace en carré estimé douze livres quatre pieces de tapisserie point dongrie dont trois pieces sont tenduës dans Lad. Chambre et Une piece qui n'a encore point servy, de quatre aune un quart la piece estimées ensemble En l'estat quelles sont y en ayant trois qui sont boucannées qui servent depuys trois ans a la somme de Cent vingt livres

Un chaslit de bois de merisier a colonnes torses a legard de celles du pied, et les autres tournées au tour ordinaire, garny d'une vieille paillasse, un lit de plume en Coitty vieil avec son traversin Un matelas de laine en toille de chanvre, Une couverte de roüan blanche de quatre points, Une Autre vieille couverte verte de Montpellier Une courtepointe dindienne simple Un tour de lit avec Le dossié A pant de Sarge de Caën verte, Le ciel de lit de cadis vert avec trois Verges de fer Le tout
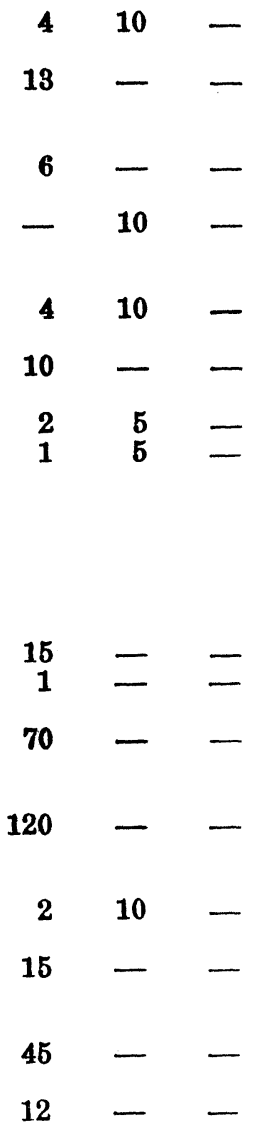
estimé Ensemble a La somme de deux cents cinquante livres cy

Deux Rideaux de fenestre de toille de lin large de Cinq aunes piece demy Uzée avec deux verges et deux pitons de fer le tout estimé neuf Livres cy

[p. 8]

Deux bouteilles de verre d'Une pinte piece, et deux autres de chopine et six verres Le tout estimé ensemble a quatre livres dix sols attendu que les bouteilles sont de verre fort

Suit ce qui Sest trouvé dans une autre Chambre qui a veuë sur la ruë derriere celle cy dessus;

Un moyen poisle de fer a chauffer avec Son Tuyau de fer de Tolle de douze feüilles ou bouts de tuyaux neuf fait de l'année derniere et estimé ensemble led poisle sans Trepied [estimé] Cent trente livres

Un moyen Lit Consistant en un bois de Couchette de bois de pin garny d'une paillasse d'un Moyen lit de plume avec Son traversin en vieux Coity, un matellas de laine en toille dechanvre avec Son tour de lit de sarge de Caen Jaune, dossier et ciel de lit et bonne grace Une vieille petite courtepointe piquée de toille Indienne, une vieille couverte blanche et deux linceux de toille de chanvre demy Uzées le tout estimé estant vieux et uzé a la somme de quatre vingt dix livres cy Une autre petite Couchette de bois de merisier avec une vieille paillasse, Un petit lit de plume en toille de chanvre deux vieilles couvertes blanches, un petit linceul un petit Traversin et un vieux pavillon de vieille sarge de Caën grise Le tout estimé a Vingt cinq livres cy

$\mathrm{Na}$ esté fait aucune prisée et estimation d'un vieux coffre bahu et d'une petite armoire de deux pieds de large sur trois pieds de haut a un seul batan fermant a clef de bois de pin attendue quils Servent et sont destinez pour Le service desd. mineurs et pour Reserver leurs hardes;

Ne sera non plus fait aucun Inventaire des Livres, habits et linges et autres Choses a l'usage de Mond Sieur Le lieutenant general attendu quils luy sont reservez par son contract de mariage passé Entre Luy et lad. deffunte dame lambert par deffunt bequet notaire Royal en cette prevosté en datte du 28. decembre 1677. Cy apres Inventorié Soûs la Cotte B, et non plus que des habits linges hardes et autres choses a l'usage desd mineurs dont mond. sieur le lieutenant general n'a voulu quil fust fait aucune prisée;

Trois petites Chezes de bois dont deux garnies de paille et une en fauteüil estimées ensemble quatre livres cy

[p. 9]

Suit ce qui sest trouve dans le grenier de lad. maison;

deux minots de bled dinde a Six livres le minot

250

9 
Un minot de bled francois estimé six Livres

Environ Cent Soixante dix Livres de farine de france estimée vingt cinq livres cy

Une grosse paire de panture a porte et vingt petits verroüx de fer avec une mechante paire de petites (un mot illisible) [?] qui ne sont propres qu'en ferrailles le tout ensemble estimé a La somme de douze livres cy Un meschant petit lit de plume de volaille en vieille toille toute uzée et persée estimé avec deux mechantes oreillers a huit Livres cy

Huit petites oreillers couvertes de toille dolonne a mettre sur des sieges estimez a dix sols piece

Une peau de caribou passée en blanc estimée 6 livres Ne sera fait aucune prisée de vingt trois madriers de pin attendu quils seront compris dans ce quy Sera cy apres declaré alegard de la batisse de la maison;

Six vieux fuzils dont les montures sont delabrées quatre denviron trois pieds de Canon et deux autres d'environ quatre pieds et demy de canon estimez ensemble avec un vieux Canon de fuzil a la somme de Cinquante Livres Une vieille piece de tapisserie de bergame denviron quatre aune Estimé cinq livres cy

Un petit vieux coffre de bois de pin fermant a clef avec une serrure platte estimé quarante sols

Un arosoïr de jardin de cuivre rouge estimé douze livres Un moule de bois a chandelle estimé quarante sols Une vieille chaudiere ronde d'environ un Seau qui n'est propre que pour mitraille estimée trente sols

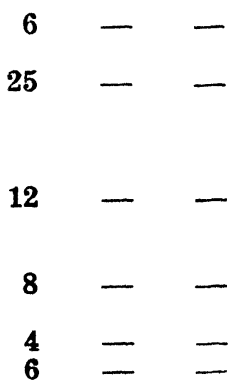

Dans le Cabinet de mon dit Sieur le lieutenant general quy a veüe sur la Cour sest trouvé;

Une couchette de bois de merisier neuve une paillasse, [p. 10]

un petit Lit de plume En toille une couverte rouge de normandie demy uzée Estimée avec une autre couverte blanche aussy de normandie demye uzee et un moyen matelas de laine en toille que mond. Sieur Le lieutenant general a declaré que mademoizelle de lotbiniere l'une de ses mineures a porté a son voyage de montréal dont il a depeint la qualité, a la somme de cinquante huit Livres avec un petit oreiller cy

declare Encor mond Sieur le lieutenant general quil y a au Seminaire de cette ville un petit matellas et Une Couverte pour le service d'un desd. mineurs quy y est pensionnaire quil dit pouvoir valoir ensemble la somme de vingt livres cy

plus Chez Les Reverends peres Jesuittes un petit matelas et une couverte pour le service d'un autre desd. mineurs quy y est aussy pensionnaire quil a aussy dit pouvoir valoir lad somme de vingt livres

Douze Ceüilleres et douze fourchettes, Une escuelle a oreilles et deux petites salieres et deux autres petites Ceüilleres d'enfant Le tout dargent pezant ensemble neuf marcs deux onces a quarante cinq livre le mar soit la somme de 
Six couteaux de table a manche noir estimez trois livres dix sols

Une pelle beche et un bechon pour le jardin Edtimez ensemble quatre livres dix sols

Suit ce qui C'est trouvé dans la Cave de lad. maison;

Une barrique de vin Rouge (un mot illisible) de bourdeaux estimée a quatre vingt dix Livres cy

Une autre barique les deux tiers vide Estimée trente livres

Deux barriques vides a quarante sols piece

Un petit bluteau a farine avec son coffre et son arbre à Trois Toilles degarny de ses Toilles estimé seulement a dix livres attendu quil Le fault demonter pour le transporter d'un mechant grenier decouvert ou il est dans un autre grenier

Ne sera fait aucune estimation d'un vieux tableau de la sainte vierge attendu que son cadre est de bois uny de peu de consequance

Tous les quels meubles cy dessus Inventoriez au present inventaire sont demeurez en la garde et possession de Mond. Sieur Le lieutenant general quy S'en est volontairement chargé du consentement dud. Sieur de grand[p. 11]

ville subrogé tuteur; a la charge par luy de les Representer ou leur valeur et d'en rendre compte toutes fois et quantes a quy il apartiendra; Et Remis la continuation dud Inventaire pour les papiers et autres effects depandant de lad Communauté a demain Sept heures du matin; et ont mond. sieur Le lieutenant general Led Sieur de grand'ville, prieur huissier priseur, et notaires Signé,
R Chartier
de Lotbinière
De Granville
Prieur
Rageot
Chambalon

Et advenant Le seiziesme jour dud. mois de Juin et an Sept cent un huit heures du matin a esté par Les Notaires Royaux susd. et soussignez ce requerant Mond Sieur Le lieutenant general Led Sieur de grandville present proceddé a la Continuation dud. Inventaire ainsy quil Ensuit;

[Decl] Suit ce qui S'est trouvé dans Le Cabinet de mond. sieur Le Lieutenant general qui a veuë sur la Cour;

En argent monnoyé et monnoye de Cartes La somme de trois Cents dix huit Livres neuf sols quatre deniers cy

Suivent les dettes actives de lad. Communauté deuës par les cy apres nommez Comme il paroist sur la liste de Comte que tient Mond. Sieur Le lieutenant general relié en parchemin Sans Cotte ny N. ${ }^{\circ}$ Commencée en l'année 1686. Sçavoir, 
Par Joseph lemay dit delorme a folio 2. la somme de trente livres cy

Par Jean baudet de lotbiniere a folio verso 4. la somme de quatre livres

Par Louis Montenu La somme de dix huit livres a folio verso 7.

Par les héritiers de Moyse Faûre dit Saint vivien a folio recto 8. la somme de douze livres cy

Par Noël barrabé a folio verso 9. La somme de quatorze livres

Par Ignace Lemay a folio verso 10. La somme de quarante six livres dix sols cy

Par Pierre Lemay a folio Recto 11. la somme de Cinq Livres cy

[p. 12]

Par Mathurin Gregoire a folio recto 13. la Somme de quarante deux Livres cy

Par Jean Chastenay dit laguine a folio recto 14. la Somme de dix Livres

Par Jean danios a folio recto 16 . la somme de treize livres

Par Jean denevers a folio recto 22. la somme de six livres

Par daniel Denevers a folio verso 24. La somme de vingt sept Livres dix sols

Par Gaspard Rérou dit Laliberté a folio recto 26. la somme de trente livres

Par René Reaume a folio verso 28. La somme de deux cent Livres cy

Par Jean barrabé fermier du domaine de lobiniere a folio verso 32 . La somme de deux Cens vingt Cinq Livres un sol huit deniers

Par Monsieur de vitré conseiller au conseil souverain de ce pays La somme de dix sept livres pour une année de rente fonciere escheuë le premier avril dernier cy

Par les heritiers ou Representants françoise hurault pour deux années de rente fontiere [escheuë] qui échoiront le 24. de ce mois La somme de quatre livres cy

Par Le sieur guillaume Gaillard marchand la somme de Cent Livres

Par Monsieur [de] Aubert de la Chenaye conseiller au Conseil souverain de ce pays par son billet du 18 . decembre 1696. payable au vingtiesme octobre de lannée suivante la somme de trois mille trois Cens livres monnoye de france, qui fait monnoye de ce pays Celle de quatre mil quatre Cens Livres Sur quoy Mond Sieur Le lieutenant general a declaré avoir Reçu dud sieur de la chenaye par les mains

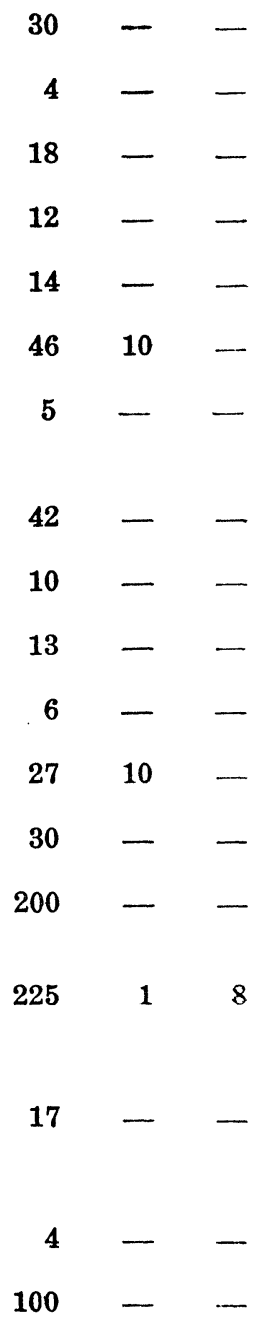


du Sieur Jean Gobin marchand la somme de trois Cens livres et ainsy reste que doit mond Sieur De la chenaye monoye de ce pays La somme de

Par Monsieur de la durantaye, pere, la somme de vingt livres

Suivent Les dettes passives de lad. Communauté; qui Sont duës aux cy apres nommez Sçavoir;

A dame françoise Jachée cy devant veuve de antoine Gourdeau vivant Sieur de beaulieu, presentement se[p. 13]

conde Epouse de Mond. Sieur le lieutenant general des Il y a plusieurs années et des le vivant de lad. deffunte dame lambert sa première Epouse dont lad. dame Jachée a un billet; la somme de deux Cents [cinquante] quatre vingt treize livres dans laquelle La valeur du dit billet est comprise cy

a Guillaume pagé dit Carcy Environ la somme de Cent vingt Livres avec Lequel il a compte a faire et Croit quil Luy doit quelque chose de plus

A la damoizelle veuve du sieur pachot Environ La somme de Soixante quinze livres avec laquelle il a Compte a faire cy

$\mathrm{Au}$ sieur françois hazeur Marchand de cette ville Environ la somme de Cent vingt livres avec Lequel il a aussy Compte a faire cy

$\mathrm{Au}$ sieur delino marchand tant en son nom que comme marguillier de la paroisse de Cette ville La somme de deux cent cinquante livres ou Environ, avec lequel il a aussy Compte a faire cy

$\mathrm{Au}$ sieur bouteville marchand environ la somme de trente livres avec lequel Il a aussy Compte a faire cy Au sieur Soulard arquebuzier du Roy Suivant son memoire de Compte quil a fourny la somme de quatre vingt six livres

Aux Dames Religieuses Ursulines de cette ville pour pention des mineures la somme de deux cents livres ou Environ cy

A Joseph maillou M.e masson Environ La Somme de Cent trente livres cy

Aux dames Religieuses hospitalieres la somme de Cinquante livres pour une année de la rente de mil livres de principal a Constitution escheuë le mois de mai dernier cy

A la fabrique de l'eglize parroissialle de quebec pour deux années de la rente de son banc escheuës a pasques dernier La somme de seize livres cy

A Pierre Maufet Charetier Environ Cinquante livres avec Lequel Il a aussi Compte a faire cy

Aux R.P. Recollets Environ La Somme de Cent Livres cy

A La dame veuve du sieur baudoüin vivant Chirurgien La somme de Cinquante livres cy

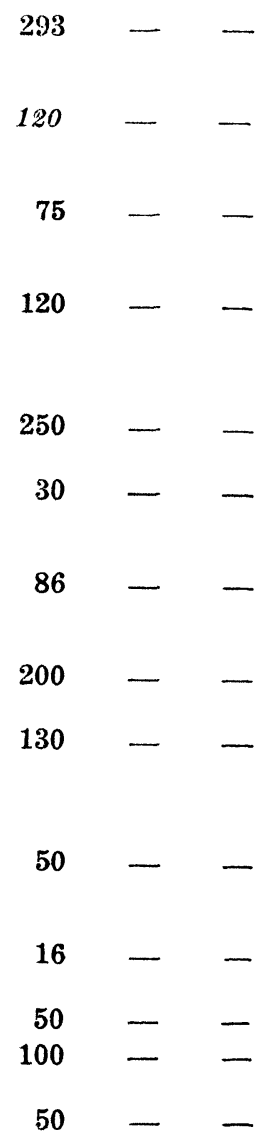


Aux heritiers de deffunte marie Varin vivante femme d'anicet boyer dit Jolicoeur par compte arresté peu de temps avant Sons deceds la somme de quarante neuf Livres cy

Au sieur Testar de Montigny lieutenant des trouppes Entretenuës en ce pays la somme de vingt Livres cy

[p. 14]

Au sieur Martel La somme de cinquante Cinq Livres cy A Chambalon l'un des notaires soûs signez suivant Le Compte au menu quil en a Soumis a Mond. sieur le lieutenant general dud jour 16 . Juin 1701. la somme de soixante Livres six sols six deniers cy

A baudet dit Lafleur boucher la somme de Cinquante livres

A michel Cadet boucher La somme de trente livres ou Environ

Declare Mond. sieur le lieutenant general quil y a au domaine de Sa seigneurie de lotbiniere deux boeufs de quatre ans qui Luy ont Couté soixante dix Livres La piece faisant ensemble

Trois vaches a trente livres La piece faisant Ensemble quatre cochons Norritureaux estimez a Cinq livres la piece

Douze poulles et un cocq estimez ensemble a six Livres dix sols cy

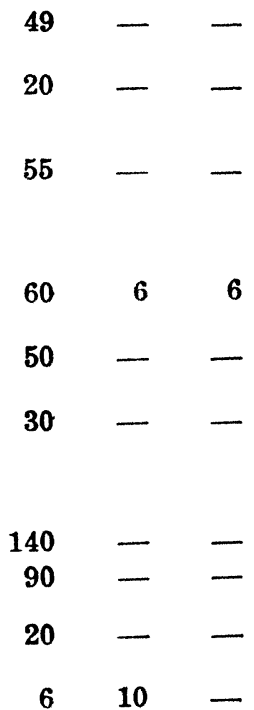

Declare Encor mond. sieur le lieutenant general quil a depansé pendant la cours de lad. Communauté et deux dernieres d'ycelle la somme de trois mil livres a faire construire Le moulin a eau, deux maisons de piece sur piece couvertes de planches Une sur le domaine de vingt trois pieds de long sur vingt pieds de large; et Lautre aud. moulin denviron vingt cinq pieds de long sur vingt de large et deux granges l'une sur led. domaine, et l'autre sur la terre quy depend dud. moulin; Lesquelles maisons sont vieilles aussy bien que lesd. granges dont Celle dud. moulin tombe en ruine, Tous lesquels [depand] bastiments Mond sieur le lieutenant a declaré estre diminuez de valeur a present de plus de moitié par leur vieillesse en attendant quil soit en commodité d'en faire faire lestimation et de quelques Ustancilles servant au labourage qui sont sur Led. lieu;

Declare aussy quil n'a depensé Jusques a ce Jour que la somme de six mil livres a faire bastir la maison de massonne en mansarde qui est presentement sur son Emplacement de Cette haute ville sise ruë Saint louis de quarante cinq pieds et demy de long sur trente quatre pieds de large a un Estage de massonne et la mansarde massonnée, attendu lavantage quil a eu de faire tirer la pierre pour la batisse d'ycelle sur le lieu, et lepargne de massonne que le roché fait du Costé du Jardin et aux deux pignons; dans laquelle maison Il reste a faire toutes les cloisons de la mansarde et Le planché du grenier qui est au dessus d'ycelle, a la reserve de la Cloison

[p. 15]

du tambour de lad. mansarde qui est faite; de maniere quil reste Lesd. Cloisons de mansarde et led planché dud. grenier a faire, pour raison de quoy Il conviendra acheter Les madriers Clouds, façon douvriers et autres 
choses necessaires a la reserve de trente madriers qui sont dans Lad. mansarde dont Il est cy devant parlé, qui sont compris en la dépanse cy devant faite et quy sont destinez a y estre employez;

Declare encore quil reste a faire dans lad mansarde tous les chassis sur douze croizées qui y sont et qui ne sont presentement fermées que par des bouts de planches; et deux Cheminées quil y doit faire construire; Ce qui a esté veu et Examiné par Led. Sieur de grandville en presence desd. notaires Soûs signez;

Suivent Les titres, papiers et Enseignements depandant de lad. Communauté paraphes desd. Notaires Soûs signez et Cottez ainsy quil Ensuit;

Un acte en papier par lequel mond. Sieur le Lieutenant general a esté esleu tuteur a sesd Enfants mineurs, et led. Sieur de grandville leur subrogé tuteur Expedié pardevant Monsieur Le lieutenant particulier de cette ville en datte du neuf du present mois de Juin et an, paraphé et Cotté A. Une grosse en parchemin du Contrat de mariage passé entre Mond. Sieur Le lieutenant general et Lad. deffunte dame Magdeleine Lambert Sa premiere espouse par deffunt M.e Romain becquet vivant notaire Royal en Cette prevosté en datte du 28. decembre 1677. paraphée et Cottée

B.

Un Extrait en papier par lequel Il parroist que Mond. sieur le lieutenant general a esté baptisé Soûs les noms de René Louis Charetier fils de louis Theandre Chartier escuyer et de damoizelle élizabeth d'amours Sa femme en leglize parroissialle de Saint Nicolas dechamps a paris Le Jeudy quatorze novembre 1641. expedié et Signé J.L. Joly vicaire de lad. Eglise du premier Juin 1675. paraphé et Cotté

Un titre de Concession en papier fait par Monsieur de Lauzon cy devant gouverneur et lieutenant General en ce pays a denis duquet d'un demy arpant de terre dans lanclave de quebec proche sa maison tenant d'un bout sur la grande routte allant au Cap rouge, par le derriere sur le chemin du mont carmel moyennant six deniers de cens en datte du 1.er Juillet 1655. Signé de lauzon et plus bas par monseigneur Roüer et, scellé du cachet de ses armes en cire d'espagne Rouge paraphé et Cotté

Un autre Titre de Concession en papier fait par monsieur du bois davaugour Aussy Cy devant gouverneur et lieutenant general en ce pays aud. denis

[p. 16]

duquet de ce qui reste de terre entre led. duquet et Louis fontaine borné par Le haut du Chemin qui va du fort au mont Carmel et par le bas a la grande allée, et de plus ce qui est entre Led. duquet et Jean Lenormand a la charge aussy de six deniers de cens, en datte du 20.e Juillet 1663. signé dubois davaugour et scellé du Cachet de ses armes en cire despagne Rouge paraphé et cotté

E.

Un Contrat dechange fait (En parchemin) 1 entre deffunt Monsieur M.e Louis Theandre Chartier escuyer sieur de lotbiniere Conseiller du Roy en ses Conseils lieutenant general civil et Criminel de cette prevosté et Les Reverendes dames Religieuses hospitalieres de lhostel dieu de cette ville en datte du 27. may 1671. paraphé et Cotté

F.

Un acte de donation en parchemin fait par monsieur Louis Chartier escuyer sieur de lotbiniere cy dessus nommé et dame Elizabeth damours son espouze

1 Cette parenthèse est de Chambalon. 
a mond. sieur Le lieutenant general sous le nom de monsieur M.e René louis Chartier Escuyer et sieur dud. lieu de lotbiniere (leur fils) 2 faisant alors les fonctions de procureur general du Roy au Conseil souverain de ce pays passé par Led. feu sieur bequet Notaire en datte du premier avril 1674. enregistré aud. Conseil souverain du neuf du mesme mois et an Cotté G.

Une Grosse en parchemin d'un Contrat de bail a rente fontière non racheptable a monsieur M.e Charles denis Escuyer sieur de vitré conseiller du roy au Conseil souverain de ce pays et a dame Catherine de Chastelnau son epouse d'Un Emplacement de trente huit pieds neuf poulces de front sis sur la rüe de la grande allée, presentement nommée Rüe saint louis; sur trente huit pieds et demy de proffondeur, a la charge de dix sept livres de rente annuelle et perpétuelle passé par led. bequet notaire en datte du 28. aoust 1674. et Cotté

$\mathrm{H}$.

Une Grosse En parchemin d'un Tiltre de Concession fait par Monsieur de montmagny $\mathrm{Cy}$ devant Gouverneur et lieutenant general pour le roy en ce pays a françois de Chavigny escuyer sieur dud. Lieu de deux arpants de Terre ou environ En datte du quatorze Juin 1647. au bas duquel est La Copie de l'acte de confirmation faite par Messieurs de la Compagnie dud. pays datté à paris en leur bureau du 29. mars 1649: Un contract de vente fait par Jacques Gourdeau Escuyer sieur de beaulieu a mathieu huboust sieur de lonchamp de quarante deux perches de terre moyennant La somme de trente deux livres passé par audoüart no.re en datte du 29. octobre 1660 . Un autre acte de declaration fait par led. sieur Jacques Gourdeau et damoizelle Eleonnard de grand maison sa femme au proffit de deffunt Led. [p. 17]

sieur louis Theandre Chartier en datte du 10. novembre 1662 passée par led. audoüart; et un autre [acte passé] declaration passée par Led. sieur Gourdeau et lad. damoizelle de grand maison sa femme Le mesme Jour par Led. audoüart notaire Cottées

Une grosse du Contract de vente en parchemin fait par Monsieur M.e louis Theandre Chartier escuyer sieur de lotbiniere a Mond. sieur René Louis Chartier (cy dessus denommez) 3 de quatre livres seize sols de rente fontiere non rachetable passé par le dit sieur bequet notaire en datte du douze avril 1674. paraphé et Cotté

L.

Un arrest du Conseil Souverain de ce pays portant Condamnation de dix livres de [Rentes] Cens et rentes fontieres seigneurialle alencontre de louis asselin au proffit de mond. Sieur le lieutenant general en datte du deuxiesme may 1695. paraphé et Cottée

Une Grosse d'obligation consentie par Jacques Renaud et Marie Charier sa femme au proffit de damelle marie laurance veuve du Sieur Estache lambert de la somme de trois cents soixante livres passée par deffunt M.e pierre duquet notaire royal en datte du $10 .^{e}$ mars 1683 . au bas de laquelle est Le Reçu de Mond. sieur le lieutenant general de la somme de Cent quatre vingt Livres en datte du 6. febvrier 1696. Cottée Un Contract 4 de vente fait par mond. S.r Le lieutenant général Et lad. deffuncte dame son Espouse a Guillaume Pagé dit Carcy d'un emplacement sis en cette basse ville rüe S.t Pierre de Cinq.te neuf pieds de front sur soixante dix de proffondeur, a la reserve de trente pieds de long sur six

2 Cette parenthèse est de Chambalon. Ragot.

3 L'écriture jusqu'ici était celle de Chambalon. Commence celle de

4 Cette parenthèse est de Chambalon. 
de large pour le prix et somme de mil Cinq.te livres passé devant Genaple no.re le douze 7.bre 1695 Cotté

Un desistement de bail a ferme passé entre mondit sieur le Lieutenant général et Noel barabé devant led. sieur Genaple no.re en datte du $89^{\text {bre. }}$ 1695 Cotté

Un Contract de Concession [pas] fait par mondit S..$^{r}$ le lieutenant général a Jean bodet de dix arpants trois perches de terre de front sur trente de proffondeur passé par Deffunct S.r Gilles Rageot aussy no.re le 2. mars 1680 Cotté

Un acte d'allignement de la Closture de la Cour et maison de mon dit sieur le lieuten..$^{t}$ général seize en Cette haute Ville rûe $s^{t}{ }^{t}$ Loüis fait par led. S.r Genaple Commis de mons. ${ }^{x}$ le grand voyer en ce pays en datte du 20.e Juillet 1699 Cottée

Deux quittances jointes signées Roüer Villeray pour Arrerages de Cens et Rentes en datte des 269 .bre 1693 et 25. 9. bre 1700 Cottées $\mathbf{S}$ Une quittance signée Peuvret a mad.me Dailleboust pour arrerages de Rentes en datte du 13. avril 1669. Cottée

[p. 18]

Une Grosse de Contract de Vente en papier fait par Denis Duquet et anne Gautier sa femme a dame marie barbe de Boulogne Veufve de deffunct Messire Loüis dailleboust cy devant gouverneur général en Ce pays d'une maison et estable et jardain Contenant un demy arpant de terre Seize en Cette haute Ville sur La rüe nommée presentem.t rue S.t Loüis moyennant la somme de deux mil Livres passé par deffunct Duquet aussy no. ${ }^{\text {re }}$ le dernier Juin 1665 [Cottée] au bas duquel sont deux quittances [V] de lad. Vente du 15 Juillet 1665 et 6 7. bre 1666 par Collation faite par led. deffunct $s^{\mathrm{r}}$ Becquet no.re le 14. aoust 1671. Cotté

Un Contract de Vente fait par marie magdelaine Brossard femme de louis fontaine a lad. Dame dailleboust passé par ledit sieur Becquet en datte du 6 Juillet 1666. Cotté

Une Coppie Collationnée de procura. on donnée [par le] au s. ${ }^{\mathbf{r}}$ pierre Peiré par le s. ${ }^{\mathbf{x}}$ Thomas bertrand mar.d bourgeois de paris passée Par Crobin et cronier no.res royaux a Hennebon le dix avril 1695, lad. Collation faite Par led. S. Rageot no.re le dix octobre mil six Cent quatre Vingt Seize Cottée

Une décharge de plusieurs Papiers que led. s. ${ }^{\mathrm{r}}$ Bertran a donnée a mond. s. $^{\mathrm{r}}$ le lieutenant général sous seing Privé en datte du Premier octobre 1698 Cotté

Un acte de foy et omage Rendû par mond. S.r le lieutenant général A mons.r Duchesnau cy devant yntendant en ce pays a Cause de sa Seigneurie de lotbiniere en datte du 12 juin 1677 Cotté

Un aveu et dénombrem. ${ }^{t}$ fait par mond. $S .^{r}$ le lieuten. ${ }^{t}$ général a cause de sa seigneurie de Lotbiniere passé devant led. Becquet notaire en datte du 3 7.bre 1677 Cottée

Un tiltre de Concession en papier accordé par mons. ${ }^{\mathrm{r}}$ Talon cy devant Intendant en ce pays au s. ${ }^{\mathbf{x}}$ Marsollet d'une demye lieüe de terre de front sur demye Lieüe de Proffondeur a prendre sur le fleuve S.t Laurent depuis la Riviere Duchesne en tirant Vers les terres S. te Foy en fief et seigneurie a la charge de la foy et homage au Chasteau S.t Louis de Quebec et les droits et debvoirs accoustumés en datte du trois 9.bre 1672 signé Talon et plus bas par mon dit seigneur signé Varnier et a costé est le cachet des armes dud. S.r Talon en Cire despagne Rouge Cottée 
Un tiltre de concession aussy accordé par mondit S. ${ }^{\mathrm{r}}$ Talon a mon dit. S.r Le lieuten.t général de sad. terre et seigneurie de lotbiniere en datte du 6 9. bre 1672 Pareillement signé et cachetté Cotté

CC.

Un procez Verbal des bornes Posées entre lad. seigneurie de Lotbiniere et Celle de S.te Croix par Jean Guion Dubuisson arpanteur En ce pays en [p. 19]

datte du 25 aoust 1679, Cotté

DD.

Un jugem. ${ }^{t}$ sur Requeste rendû par mons. demeûle aussy cy devant Intendant en ce pays entre mond. S. ${ }^{x}$ le lieutenant général et la Veuve et heritiers du feu S. ${ }^{\mathbf{x}}$ Marsollet Portant quils feront jncessamm.t arpenter et borner leur terre en datte du 24 avril 1683 Signé demeule et plus bas par mon seigneur de Chasseur, Cotté

EE

Une donation entre Vifs faite Par le $\mathbf{s}^{\mathrm{r}}$ denis Le Maistre et marie Barbier sa femme au s. ${ }^{\mathbf{r}}$ Charles Damours Escuyer S.r de Louviere d'une terre en fief d'une demye lieüe de front sur Une lieüe et demye de proffondeur a Prendre depuis La Riviere Duchesne Jusques aux terres depuis Conceddées a mond s. $^{\mathrm{r}}$ lieutenant général Passé par led. S.r Genaple no.re en datte du 26. Janvier 1684 Insinuée a la Prévosté de cette ville le troisiéme mars en suiv.t Cottée

FF

Un Jugem. ${ }^{t}$ Rendû entre mond. s. $^{r}$ le lieuten. ${ }^{t}$ gnal et led. $S .^{r}$ de Louviere par [lequel] Mond s. ${ }^{x}$ Demeûle yntendant par lequel led. $s^{x}$ de Louviere Demeure Propriettaire de la terre cy dessous a luy Donnée en datte du 20. Mars 1685 Cottée GG

Un tiltre de concession en Parchemin fait Par messieurs lefebvre de la barre et Demeûle gouverneur gnal et intendant de ced. Pays a mond. s. le lieuten.t gnal de trois quarts de lieüe de terre de front sur deux lieües de proffondeur depuis la grande Riviere Duchesne en Remontant a la petite Riviere du même nom en datte du Premier avril 1685 Signé lefebvre de la barre, Demeûle, et plus bas Par mes seigneurs Peuvret et scellé en Cire Rouge Cotté

$\mathrm{HH}$

Une grosse de transaction Passée entre mons. ${ }^{r}$ M.e Mathieu damours Vivant Ecuyer seig. ${ }^{x}$ Dechaufour Cons. ${ }^{x}$ au Cons. ${ }^{1}$ souverain de ce pays passé devant led. S.r Rageot no.re le 22 febvrier 1686 [Signé] Cotté

II

[Un Pacquet Cachettés en cinq Endroits de Cire d'espagne Rouge sur lequel est L'Inscription qui suit;]

La ligne et demye cy dessus Raturée ne Vault; Et attendu qu'yl est six heures sonnées nous avons remis la continuation dud. Inventaire a demain sept heures du matin et sont Les choses cy dessus Inventoriées Restées es mains de mond. s. ${ }^{r}$ Le Lieutenant général qui s'en est Volontairem. ${ }^{t}$ chargé du Consentement dud. s. $^{\mathrm{r}}$ de granville subrogé Tuteur qui a avec luy et Lesd. no.res signé

\section{R.L. Chartier \\ de Lotbiniere}

Rageot
De Granville

Chambalon

[p. 20]

Et advenant le dix septie.e Jour du mois de juin et an 1701 sept heures du matin a été requerant mond S.r Le lieuten..$^{\mathbf{t}}$ gnal et led. S.r de grandville procedé a la Continuation dudit Inventaire ainsy qu'yl Ensuit.

Suitte des papiers 
Une Reconnoissance 5 en original portant quittance de la somme de douze cents livres donnée par Monsieur M.e Mathieu damours Conseiller au conseil souverain de ce pays faisant pour Charles damours Escuyer Sieur de louviere son fils a Mond. sieur le lieutenant general passé par Led. feu sieur Rageot notaire royal en datte du 22. febvrier 1682. paraphée et Cottée LL Une grosse en papier d'acte de Renonciation faite par Mond. Sieur Le lieutenant general Tant en son nom que comme procureur de dame Marie françoise Chartier veuve de pierre de Joybert escuyer Seigneur de Soulange marsan et autres lieux Commandant en laccadie, a la Succession du deffunt Monsieur M.re louis Theandre Chartier escuyer Seigneur de lotbiniere Leur pere vivant aussy lieutenant general en la prevosté de cette ville passé par Levesque et Raymond notaires au Chatellet de paris en datte du $2 .^{\circ}$ mars 1692. Cottée

MM

Une Requeste presentée par Mond. sieur le lieutenant general a monsieur le lieutenant particulier de la prevosté de cette ville par Laquelle Il Reytere la Renonciation cy dessus au bas de laquelle est Un Jugement de mond. sieur le lieutenant particulier du 20.e decembre 1695. rendu sur lad. requeste Cottée

NN.

Une quittance de Madame de Marsan soûs seing privé, par Laquelle elle Reconnoist avoir receu de mond. sieur Le lieutenant general la somme de deux cents livres de france faisant moitié de celle de quatre cents livres quil a receuë de Monsieur duval en consequance d'une transaction passée entr'eux par bonnetaire et despriot no. ${ }^{\text {res }}$ au Chatellet de paris en datte du 16. mars 1692. lad. quittance en datte du 27.e mars 1693. Signée M.f. Chartier de marsan Cottée

00 .

Un billet de Monsieur de la durantaye dont La Teneur suit, Le besoin que Jay dargent m'oblige de vous supplier très humblement de donner a ma femme les six cents livres de france moins trente sols que vous sçavez et vous obligerez fort vostre très obeisant serviteur de la durantaye a quebec ce quatre octobre 1692 . Cotté

PP.

Une quittance de Madame de la durantaye de la somme de Six cents livres [p. 21]

de france, [sur le] suivant le billet cy dessus a mond. sieur Le Lieutenant general en datte du 11.e octobre 1692. Signée françoise duquet Cottée QQ. Dix quittances des dames Religieuses hospitallieres de cette ville pour vingt années de rente de Cinquante Livres par an et ce pour Les années Echeues le 27. may 1681. Jusques et Compris l'année escheuë le 27. may 1700, lesd. quittances pour deux années chacune nombrées et paraphées depuis premiere Jusques a dixiesme et derniere et lad derniere Cottée

RR.

Un memoire et neuf lettres escrittes par Messieurs de l'ancienne Compagnie de ce pays a deffunt Monsieur M.e louis Theandre Chartier escuyer seigneur de lotbiniere vivant lieutenant general de cette prevosté les 3. may 1668., 14. mars 1670. 19. avril 1671. 19e. may 1672. 18. Juin 1673, et premier Juin 1674. aussy nombrées et paraphées depuis premiere Jusques a dixiesme (sic) et derniere et Cottee sur la derniere Seulement

Une grosse en parchemin Timbré du Contract de Mariage passé entre Mon dit Sieur louis Theandre Chartier, et damoizelle Elizabeth damours pere et mere de Mond. Sieur le lieutenant general par lorimier et nourry Notaires au Chastelet de paris, en datte du $6^{\mathrm{e}}$ febvrier 1641. par lequel Contract Lad. daz. ${ }^{l e}$ damour a ameubly le tiers de ses biens, le Douaire Reglé a quatre cents Livres de rente, et le preciput a quatre cents livres, Colla-

${ }^{5}$ Ici reprend l'écriture de Chambalon. 
tionnee par Torenon et Aumont no.res aud. Chastellet Du 16. aoust 1691. es mains duquel dit Aumont estait alors laminutte dud. Contract, Cottée TT. Des lettres de provisions de loffice de lieutenant general de la prevosté de quebec accordées en datte du 13. may 1675. par Sa Majesté a Mond. sieur louis Theandre Chartier Signées louis, et sur le reply par Le Roy, Colbert scellées en Cire Jaune, registrées au Conseil souverain de ce pays Le 23. Septembre 1675. Cottées

UU.

Une ordonnance de Monsieur dubois Davaugour cy devant Gouverneur general en ce pays en datte du 11. mars 1662. par laquelle Il ordonne qu'une lettre écritte par Sa Majesté a Mond. feu Sieur le lieutenant general pere que sera remise au greffe de la Senechaussée de quebec, qui en avoit esté ostée par ordre de Monsieur dargenson aussy cy devant Gouverneur et lieutenant general En ce pays, et quelle y seroit registrée pour lhonneur de sa famille, au bas de laquelle est écrit, Enregistrée au Registre des Insinuations du greffe avec la lettre du Roy par moy Greffier le $11^{\circ}$ mars 1662. Signé Gourdeau greffier Cottée XX. Une lettre de Monsieur larchevesque de Roüan escrite a Mond. Sieur [p. 22]

Le lieutenant general pere dont la Teneur Suit: Monsieur, Comme vous Exercez Tres dignement loffice de lieutenant general dans Le pays de La nouvelle france, Jay Crû devoir accompagner La lettre du Roy, d'une des miennes pour vous prier de tenir la main a l'execution des ordres de Sa Majesté, Monsieur labbé de quelus en causera avec vous et vous Eclaircira de toutes les Choses quy vous peuvent servir d'Instruction pour maintenir l'autoritté du pape, la mienne qui Suis ordinaire dans le pays et Celle de $\mathrm{Sa}$ Majesté avec lequel je suis convenu de toutes choses, de Surplus Monsieur croyez moy tout a vous et que dans les occazions Je vous veux témoigner avec quelle passion je suis, Monsieur, vostre Tres affectionné serviteur archevesque de Roüan, et acosté est escrit, de paris le 3. may 1659., Monsieur Chartier, et sur lenveloppe a M.r chartier Conseiller du Roy en ses Conseils et lieutenant general pour la Justice au Royaume de la nouvelle france, en Canada; Cotté YY.

Des lettres de provision de loffice de lieutenant general Civil et Criminel en la ville de quebec accordées a Mond. sieur de lotbiniere pere par Messieurs les directeurs generaux de la Compagnie des Indes occidentalles en datte du 14 mars 1670. signée brunet, bibaud, bellizany, berthellot et pelissier et plus bas par lad. Compagnie daulier; et scellé du scau de lad. Compagnie en cire rouge Cottée

$\mathrm{ZZ}$

Un arrest du Conseil souverain de ce pays par lequel Mond. sieur de lotbiniere pere a esté Receu et Installé en l'office de lieutenant Civil et Criminel dud. quebec en datte du 10. Janvier 1667. cotté W.W.

Des lettres de provision de lieutenant Colonel du regiment de quebec accordées a Mond. Sieur le lieutenant general par Monsieur le Comte de frontenac cydevant Gouverneur et lieutenant general pour le Roy en ce pays en datte du 2 e Juin 1673. Signée Frontenac et plus bas par Monseigneur le Chasseur et Scellees Cottees

AAA.

Un acte en parchemin de Nomination a loffice de conseiller au Conseil souverain de ce pays de la personne de Mond. Sieur le lieutenant general, par Messieurs de lancienne Compagnie en datte du 25. May 1674. Signé bellizany, et daulier, et plus bas par la Compagnie Daulier délandec et scelle du seau de lad. Compagnie Cotté

BBB.

Des lettres de provisions du Roy de loffice de Conseiller au Conseil souverain de ce pays accordées a Mond. sieur le lieutenant general en datte du 29. may 
1674. Signées louis et sur le Reply par Le Roy, Colbert, registrées au greffe du Conseil Souverain le 2. octobre de la mesme année et scellée en [p. 23]

Cire Jaune cottée

CCC.

Dautres lettres de provisions en parchemin de l'office de Conseiller aud. Conseil Souverain accordées par le Roy a Mond. Sieur le lieutenant general en datte du 26. avril 1675. Signée Louis, et sur le Reply Par le roy, Colbert, régistrées aud. Conseil Souverain le 23. Septembre de la mesme année Et Scellées en Cire jaune Cottées

DDD.

Une commission en papier par laquelle Monsieur du Chesneau cy devant Intendant en ce pays a Commis Mond. Sieur Le lieutenant general pour connoistre et Juger déffinitivement des differends en matiere civille qui Sont mûs et Se pourront mouvoir Entre les habitans du bout de lisle de Montreal et d'jnformer des matieres Criminelles, en datte du 16 febvrier 1677. Signée duchesneau et plus bas par Monseigneur Chevalier Cottée

EEE.

Des lettres de provisions [de loffice de lieutenant] accordées par le Le (sic) Roy à mond. Sieur le lieutenant general de l'office de Conseiller et Lieutenant general en lad. prevosté en datte du $1^{\text {er }}$ may 1677. Signée Louis et sur Le reply par le Roy, Colbert et Sellée en Cire jaune registrées au Conseil Souverain de ce pays et en lad. prevosté les 25. et 29. octobre 1677. Cottées

FFF.

Un arrest du conseil Souverain de ce pays par lequel Mond. sieur Le lieutenant general a esté receu aud. office de lieutenant general en datte du 25. octobre 1677. Cotté

GGG.

Des lettres de provision de Commandant du Regiment de quebec accordées a mond. Sieur le lieutenant general par Monsieur lefebvre de la barre cy devant Gouverneur et lieutenant general pour Le Roy en ce pays en datte du 6 Juillet 1684. Signée lefebvre de la barre et plus bas par Monseigneur Regnault et Scellée Cottée

HHH.

Un ordre en papier donné par Monsieur le marquis de denonville aussy cydevant Gouverneur et lieutenant general pour le Roy en ce pays pour Commander et faire loger les trouppes en Son absence par mond. Sieur Lieutenant general en datte du 20. may 1687. Signée Lemarquis de denonville Cottée

JJJ.

Une commission de subdelegué donnée à Mond. Sieur Le lieutenant general par MonSeigneur de Champigny, Intendant de ce pays en datte du 26. avril 1689. Signée bochart Champigny et plus bas par Monseigneur Fredin (?) Scellée en Cire despagne rouge et Cottée

LLL.

[p. 24]

Un jugement arbitral Rendu par Mond. Sieur Le marquis de denonville Mond. Seigneur de Champigny, Intendant et Mond. Sieur le Lieutenant general entre le Sieur denis Riverin et Les Interessez dans La Compagnie du nord en datte du 28.e avril 1689. Cotté

MMM.

Des lettres de provisions de Colonel de la milice en papier accordée par Mond. Sieur le Comte de frontenac, a mond. Sieur Le lieutenant general en datte du $6^{\mathrm{e}}$ Juin 1690. Signée frontenac et plus bas par Monseigneur de monseignat et Scellée en cire rouge Cottées

NNN.

Un Certificat donné par mond. Seigneur de Champigny Intendant a mond. Sieur le lieutenant general par lequel il Certifie les bons services quil a rendus en qualité de Son sudellegué, et la nécessité quil Continuë pour 
le service du Roy qui l'empesche de passer en france pour ses affaires particulieres en date du 25 novembre 1690. Signé bochard Champigny et plus bas par MonSeigneur Fredin (?) et Scellé en Cire despagne Rouge cotté

000.

Un autre Certificat de Mond. sieur le Comte de frontenac comme Il a arresté Mond. sieur le lieutenant general pour le service du Roy et Retardé son voyage de france du 25 . novembre 1690 . signé frontenac et plus bas par monseigneur de Monseignat et scellé en Cire rouge Cotté P.P.P. Une lettre de Monseigneur de pontchartrain ministre détat escrite a mond. sieur le lieutenant general dont la Teneur suit, Le Roy a esté Informé de l'integritté avec laquelle vous rendez la Justice a ses sujets en Canada et de la bonne conduitte que vous tenez dans vostre Charge, sur quoy Sa Majesté m'a ordonné de vous faire sçavoir qu'elle en est fort satisfaite et quelle vous en donnera des marques lorsquil se trouvera quelque occazion de vous faire plaisir a paris le 7.e avril 1691. Signé pontchartrain cotté

QQQ.

Un titre de Concession en papier accordé par Mond. feu sieur Le Comte de frontenac et par Mond. Seigneur de champigny Intendant a mond sieur le lieutenant general de trois lieues et demy de terre de front a prendre au bout de sa seigneurie de lotbiniere sur quatre Lieues de proffondeur a tiltre de fief et seigneurie haute moyenne et basse Justice avec droit de pesche et de chasse en datte du 25. mars 1693. Signée frontenac, et bochard Champigny, et plus bas par Monseigneur de Monseignat, par Monseigneur Peuvret et scellée du seau de leurs armes Cotté

RRR.

\section{[p. 25]}

Un brevet de Confirmation du Roy de la Concession cy dessus en parchemin en datte du 15. avril 1694. Signé louis et plus bas phelipeaux Cotté SSS. Des lettres de provisions de l'office de lieutenant general civil et Criminel de l'admirauté de cette ville accordées a mond. Sieur le lieutenant general par MonSeigneur le Comte de Toulouze admiral de France endatte du 28. decembre 1698. Signée L.A. debourbon et Sur le reply par Monseigneur devalincour, et Scellees en Cire Rouge; Cottées TTT. Une lettre Escrite par Monseigneur de pontchartrain a mond. Sieur le lieutenant general dattée a versailles du 5 may 1700. [par laquelle Il luy mande que Le Roy trouve bon quil continuë de prendre Connoissance des causes maritimes] Dont la teneur suit, A Versailles le Cinquiesme may 1700. Jay receu la lettre que vous mavez écrite le vingt quatre octobre dernier le Roy trouve bon que vous continuiez de prendre Connoissance des causes maritimes a quebec, comme vous avez fait par le passé et Je donne ordre à $M$. de Champigny de me Renvoyer la Commission qui luy a esté envoyée l'année passée pour Le sieur dupuy que Sa Majesté m'avoit fait expedier que pour confirmer la nomination faite par M.r Le Comte de toulouze, Je suis fort persuadé que vous vous acquitterez bien de cet employ, et S'il y a occazion de vous faire plaisir dans la Suitte Je Le feray volontiers Signé pontchartrain, Cottée

UUU.

Une Commission de subdelegué donnée a mond. sieur le lieutenant general par Mond. Seigneur de Champigny Intendant en datte du 25. Juin 1700 . Signée bochart champigny et plus bas par Monseigneur André Cottée XXX Declare Mond. sieur Le lieutenant general quil a Receu de Jean Guion une Somme de Cinq cents livres qui avoit esté Laissée Lors du partage de La succession de deffunte damoizelle Marie laurençay (sic) veuve de Deffunct Le sieur Estache Lambert pour estre Employée par maniere 
de fondation a faire dire et celebrer des messes pour Le Repos de lame de lad. deffunte et de ses descendants annuellement a perpetuitté, de laquelle somme Il s'est chargé, en Consequance du Refus qu'ont fait messieurs du seminaire de Cette ville d'ycelle accepter à la charge par eux de dire et Celebrer vingt cinq basses messes par Chacun an a perpetuitté n'ayant voulu accepter la proposition que Mond. sieur Le lieutenant general Leur en a faite qu'a condition quils ne feroient dire que dix huit messes par [p. 26]

Chacune années; pour raison de quoy et sur Leur Refus Il S'en Est Chargé comme dit est et a fait dire et Cellebrer Led. nombre de vingt Cinq messes par Chacune année depuis que Lad Somme a esté destinée a ce sujet et Lesquelles Il declare quil Continuera a faire cellebrer annuellement Jusques a ce que Il ayt Lieu de plasser surement Lad somme de Cinq cents Livres pour Lad. fondation;

Declare encore Mond. sieur Le Lieutenant general quil a cy devant obmis a declarer quil doit a Jacques bernier dit Jean de paris habitant du Cap saint Ignace comme Tuteur de deffunt guillaume ferté mineur Environ La somme de quatre vingt quinze livres dont Led.bernier $\mathrm{A}$ son billet et que veritablement Il n'est pas memoratif positivement de la somme pour laquelle Il est fait;

De Tous lesquels effects Inventoriez au present Inventaire que Mond. sieur le lieutenant general a dit estre tout ce qui depend de la communauté qui [Entre] a esté Entre Lad. deffuncte dame marie Magdelaine lambert sa premiere espouze et dont Il est memoratif Mond. sieur Le lieutenant general S'est volontairement chargé du conscentement dud. sieur de grand-ville subrogé auteur [pour] a la charge d'yceux representer ou leur valeur et d'en Rendre compte Toutes fois et quantes a quy Il appartiendra [et ont] fait Et arreste Led. Jour dix sept Juin mil sept cent Un et ont mond. sieur Le lieutenant general, Lesd. Sieur de grand-ville et notaires, Signé

RL Chartier

de Lotbiniere
Rageot
De Granville

Chambalon

\section{CONGRÈS}

\section{EXPERIENCES DANS L'ENSEIGNEMENT DE L'HISTOIRE}

Sous la présidence d'honneur de $\mathrm{M}$. Denis Vaugeois, directeur du Centre franco-québécois de développement pédagogique au Ministère de l'Education.

Date : samedi, le 30 mars 1968 - Lieu : Université de Montréal

Heures de 9.30 à 16.15 heures

Conférenciers : M. Michel Brunet (atelier universitaire)

M. Michel Allard

M. Gilbert Vaillancourt (atelier cegep)

Mlle Huguette Dussault
M. Jacques Archambault

$$
\text { (atelier secondaire) }
$$

M. André Lefebvre (atelier primaire)

Admission : gratuite pour les membres de la Société.

Renseignements : $\$ 1.00$ pour les non-membres.

M. Pierre Savoie,

8454 rue St-Hubert - 387-7642
M. Michel Allard

4579 rue St-André - 526-7395 